\title{
PROJECTION-ITERATION REALIZATION OF A NEWTON-LIKE METHOD FOR SOLVING NONLINEAR OPERATOR EQUATIONS
}

\author{
Liudmyla L. Hart*
}

\begin{abstract}
We consider the problem of existence and location of a solution of a nonlinear operator equation with a Fréchet differentiable operator in a Banach space and present the convergence results for a projection-iteration method based on a Newton-like method under the Cauchy's conditions, which generalize the results for the projection-iteration realization of the Newton-Kantorovich method. The proposed method unlike the traditional interpretation is based on the idea of whatever approximation of the original equation by a sequence of approximate operator equations defined on subspaces of the basic space with the subsequent application of the Newton-like method to their approximate solution. We prove the convergence theorem, obtain the error estimate and discuss the advantages of the proposed approach and some of its modifications.
\end{abstract}

Key words: nonlinear equation, Fréchet differentiable operator, Newton-like method, projection-iteration method, approximation, convergence, error estimate.

2010 Mathematics Subject Classification: 65J15, 65B99, 47A58.

Communicated by Prof. V. V. Semenov

\section{Introduction}

The fundamental tool in numerical analysis, operations research, optimization and control is Newton's method originally intended to solve algebraic equations. The basic ideas of the method, the main theoretical results of convergence, the latest developments in this area, the most up-to-date versions of the method, as well as its various applications can be found, for instance, in papers $[1,4,5$, 12-18]. Newton's method has been studied in more detail under the so-called Kantorovich conditions (the derivative of the equation operator is invertible at the initial point and satisfies the Lipschitz condition in the considered domain), under the Vertgeim conditions (the operator derivative is invertible at the initial point but satisfies only Hölder condition) and under the Mysovskih conditions (the derivative is invertible at all points in the considered domain and its inverse operator is bounded).

To solve nonlinear functional equations, other iterative methods as well as projection (approximation) type methods are also used; a survey of the relevant literature is contained, for instance, in [8]. In the same source, to solve operator

\footnotetext{
${ }^{*}$ Department of Computational Mathematics and Mathematical Cybernetics, Oles Honchar Dnipro National University, 72, Gagarin av., Dnipro, 49010, Ukraine, 11_hart@ukr .net

(C) L. L. Hart, 2019.
} 
equations of the first kind, studies have been performed for methods called the projection-iteration ones based on the following idea. An equation of the form

$$
A u=f
$$

with a nonlinear operator $A$ acting on a Banach space $X(f \in X$ is a known element), is approximated by a sequence of approximate equations

$$
A_{n} u_{n}=f_{n}, \quad n=1,2, \ldots,
$$

where $A_{n}$ is a nonlinear operator acting on a subspace $X_{n}$ of the original space $\left(X_{1} \subset X_{2} \subset \cdots \subset X_{n} \subset \cdots \subset X, \quad X_{1} \neq \varnothing\right)$. To solve approximate equations (1.2), some iterative method is used, at that for each of these equations only a few approximations $u_{n}^{(k)}\left(k=1,2, \ldots, k_{n}\right)$ are found and the last of them $u_{n}^{\left(k_{n}\right)}$ is assumed to be equal to the initial approximation $u_{n+1}^{(0)}$ in the iterative process

for the next, $(n+1)$-th approximate equation. The sequence $\left\{u_{n}^{\left(k_{n}\right)}\right\}_{n=1}^{\infty} \subset X$ is considered as a sequence of approximations to a solution $u^{*}$ of equation (1.1). This approach to finding an approximate solution of the original equation naturally eliminates the difficulties that arise when solving the same equation using the conventional projection method and also makes it easier to choose the suitable initial approximation compared to solving the original equation using the iterative method.

In this paper, to solve nonlinear operator equation (1.1), the projection-iteration implementation of the Newton-like method [6] is studied under generalized Cauchy's conditions, which instead of the inverse operator to the derivative in the considered domain imply the existence of some linear operator close to it. The problems of substantiation of the projection-iteration schemes of both the basic Newton-Kantorovich method under such conditions and some of its modifications are considered.

\section{Preliminaries}

Let us consider equation (1.1) $A u=f$ with a nonlinear operator $A$ which acts on a Banach space $X$ and is Fréchet differentiable on some ball $S\left(u_{N}^{(0)}, R\right)=$ $\left\{u \in X:\left\|u-u_{N}^{(0)}\right\| \leq R\right\}$ of this space. We approximate equation (1.1) by the sequence of approximate equations (1.2) $A_{n} u_{n}=f_{n}, \quad n=1,2, \ldots$ with nonlinear operators $A_{n}$, each of which acts on the respective subspace $X_{n} \subset X$ and is Fréchet differentiable on the set $\Omega_{n}=X_{n} \cap S\left(u_{N}^{(0)}, R\right)$ beginning with some number $n=N \geq 1 ; f_{n}=P_{n} f, P_{n}$ is a linear projector which maps $X$ onto $X_{n} \quad\left(P_{n}: X \rightarrow X_{n}, \quad P_{n} u_{n}=u_{n}\right.$ for $\left.u_{n} \in X_{n}\right)$.

Assume that for each $n \geq N$ the following proximity conditions hold:

$$
\begin{gathered}
\left\|A_{n} u_{n}-P_{n} A u_{n}\right\| \leq \alpha_{n}, \quad\left\|A_{n}^{\prime}\left(u_{n}\right)-P_{n} A^{\prime}\left(u_{n}\right)\right\|_{X_{n} \rightarrow X_{n}} \leq \alpha_{n}^{\prime}, \quad \forall u_{n} \in \Omega_{n} \\
\left\|P_{n} A u-A u\right\| \leq \beta_{n}, \quad\left\|P_{n} A^{\prime}(u)-A^{\prime}(u)\right\|_{X \rightarrow X} \leq \beta_{n}^{\prime}, \quad \forall u \in S\left(u_{N}^{(0)}, R\right)
\end{gathered}
$$




$$
\left\|P_{n} f-f\right\| \leq \gamma_{n}, \quad \forall f \in X,
$$

where $\alpha_{n}, \alpha_{n}^{\prime}, \beta_{n}, \beta_{n}^{\prime}, \gamma_{n} \rightarrow 0$ when $n \rightarrow \infty$. We will also assume that the derivative $A_{n}^{\prime}\left(u_{n}\right)$ on the set $\Omega_{n}$ satisfies the Lipschitz condition

$$
\left\|A_{n}^{\prime}\left(u_{n}\right)-A_{n}^{\prime}\left(v_{n}\right)\right\|_{X_{n} \rightarrow X_{n}} \leq L^{\prime}\left\|u_{n}-v_{n}\right\|, \quad \forall u_{n}, v_{n} \in \Omega_{n}, \quad n \geq N,
$$

where $L^{\prime}>0$ is a Lipschitz constant.

If there exists a continuous linear operator $\Gamma_{n}\left(u_{n}\right)=\left[A_{n}^{\prime}\left(u_{n}\right)\right]^{-1}$ for all $u_{n} \in$ $\Omega_{n}(n \geq N)$ then one can apply the Newton-Kantorovich method [14] to each of equations (1.2) beginning from the number $n=N$, and construct a sequence of approximations to the solution $u^{*}$ of equation (1.1) by the formulas

$$
\begin{gathered}
u_{n}^{(k+1)}=u_{n}^{(k)}-\left[A_{n}^{\prime}\left(u_{n}^{(k)}\right)\right]^{-1}\left(A_{n} u_{n}^{(k)}-f_{n}\right), \quad k=0,1, \ldots, k_{n}-1 ; \\
u_{n+1}^{(0)}=u_{n}^{\left(k_{n}\right)}, \quad n \geq N ; \quad u_{N}^{(0)} \in \Omega_{N} \subset X .
\end{gathered}
$$

In paper [3] the theorem is given on the existence of a solution $u^{*}$ to equation (1.1), on the domain of its location, as well as on the convergence of projectioniteration process (2.5) under the Cauchy-type conditions. The following theorem is a generalization of the mentioned theorem, when instead of operators

$$
\Gamma(u)=\left[A^{\prime}(u)\right]^{-1}, \quad u \in S\left(u_{N}^{(0)}, R\right)
$$

and $\Gamma_{n}\left(u_{n}\right)=\left[A_{n}^{\prime}\left(u_{n}\right)\right]^{-1}, u_{n} \in \Omega_{n}(n \geq N)$, it is required the existence only of an operator $D(u), u \in S\left(u_{N}^{(0)}, R\right)$ in $X$ and an operator $D_{n}\left(u_{n}\right), u_{n} \in \Omega_{n}$ in $X_{n}$, which are close to $\Gamma(u)$ and $\Gamma_{n}\left(u_{n}\right)$ respectively.

Theorem 2.1. Let the operator $A$ be Fréchet differentiable on some ball $S\left(u_{N}^{(0)}\right.$, $R) \subset X$ and let for all $n \geq N$ the operator $A_{n}$ be Fréchet differentiable on the set $\Omega_{n}=X_{n} \cap S\left(u_{N}^{(0)}, R\right)$, at that let its derivative $A_{n}^{\prime}\left(u_{n}\right)$ satisfy on $\Omega_{n}$ the Lipschitz condition (2.4). Assume that the proximity conditions (2.1)-(2.3) hold true and there exist a linear operator $D(u)$ on $X$ and linear operators $D_{n}\left(u_{n}\right)$ on $X_{n}$ such that

$$
\begin{gathered}
\|D(u)\|_{X \rightarrow X} \leq b, \quad\left\|E-D(u) A^{\prime}(u)\right\|_{X \rightarrow X} \leq \delta<1, \quad \forall u \in S\left(u_{N}^{(0)}, R\right) \\
\left\|E-D_{n}\left(u_{n}\right) A_{n}^{\prime}\left(u_{n}\right)\right\|_{X_{n} \rightarrow X_{n}} \leq \delta_{n}<1, \quad \forall u_{n} \in \Omega_{n}, \quad n \geq N
\end{gathered}
$$

where $b>0, \delta>0, \delta_{n}>0 ; E$ is an identity operator on $X$. If the initial approximation $u_{N}^{(0)} \in \Omega_{N}$ satisfies the conditions

$$
\left.\| A_{N} u_{N}^{(0)}-f_{N}\right) \| \leq \eta_{N}^{(0)}, \quad h_{N}^{(0)}=b_{N}^{2} L^{\prime} \eta_{N}^{(0)}<2, \quad r_{N}=b_{N} \eta_{N}^{(0)} G_{N} \leq R,
$$


where

$$
\begin{aligned}
b_{N} & =b /\left(1-b\left(\alpha_{N}^{\prime}+\beta_{N}^{\prime}\right)-\delta\right) \\
G_{N} & =H_{N}+\sum_{m=N}^{\infty}\left(h_{N}^{(0)} / 2\right)^{2^{S_{m}}-1}<2 H_{N} \\
s_{m} & =\sum_{i=N}^{m}\left(k_{i}-1\right) \\
H_{N} & =\sum_{m=0}^{\infty}\left(h_{N}^{(0)} / 2\right)^{2^{S_{m}}-1}
\end{aligned}
$$

then equation (1.1) has in the ball $S\left(u_{N}^{(0)}, r_{N}\right)$ a solution $u^{*}$ to which the based on Newton's method process (2.5) converges with the error estimate

$$
\left\|u_{n}^{\left(k_{n}\right)}-u^{*}\right\| \leq b_{N} \eta_{N}^{(0)} V_{n}\left(h_{N}^{(0)} / 2\right)^{2^{S_{n}}-1}, \quad n \geq N,
$$

where

$$
V_{n}=\sum_{m=0}^{\infty}\left(h_{N}^{(0)} / 2\right)^{2^{S_{n}}\left(2^{m}-1\right)}+\sum_{m=n+1}^{\infty}\left(h_{N}^{(0)} / 2\right)^{2^{S_{m}}-2^{S_{n}}}<2 H_{N} .
$$

The proof of Theorem 2.1 can be found in [3].

\section{Proving the convergence theorem}

Let us consider, to solve the operator equation (1.1), a projection-iteration process, like (2.5) with the replacement of the operator $\Gamma_{n}\left(u_{n}^{(k)}\right)=\left[A_{n}^{\prime}\left(u_{n}^{(k)}\right)\right]^{-1}$ by an operator $D_{n}\left(u_{n}^{(k)}\right)$ close to it:

$$
\begin{gathered}
u_{n}^{(k+1)}=u_{n}^{(k)}-D_{n}\left(u_{n}^{(k)}\right)\left(A_{n} u_{n}^{(k)}-f_{n}\right), \quad k=0,1, \ldots, k_{n}-1 ; \\
u_{n+1}^{(0)}=u_{n}^{\left(k_{n}\right)}, \quad n \geq N ; \quad u_{N}^{(0)} \in \Omega_{N} \subset X .
\end{gathered}
$$

The following theorem establishes the sufficient conditions of feasibility and convergence in the ball $S\left(u_{N}^{(0)}, R\right)$ of the approximations sequence $\left\{u_{n}^{\left(k_{n}\right)}\right\}_{n=N}^{\infty} \subset X$ determined by formulas (3.1) to a solution $u^{*}$ of equation (1.1).

Theorem 3.1. Let all the conditions of Theorem 2.1 hold true and let, moreover, the derivative $A^{\prime}(u)$ satisfy on $S\left(u_{N}^{(0)}, R\right)$ the Lipschitz condition

$$
\left\|A^{\prime}(u)-A^{\prime}(v)\right\|_{X \rightarrow X} \leq L\|u-v\|, \quad \forall u, v \in S\left(u_{N}^{(0)}, R\right) ; \quad L>0 .
$$

Assume that $b L \delta /(1-\delta)<1$, where $b>0, \delta>0$ are defined in (2.6), and that $\delta_{n} \rightarrow 0$ in condition $(2.7)$ when $n \rightarrow \infty$. If the initial approximation $u_{N}^{(0)} \in \Omega_{N}$ satisfies the first condition (2.8),

$$
h_{N}^{(0)}=b_{N}^{2} L^{\prime} \eta_{N}^{(0)}+\frac{2 b_{N} L^{\prime} \delta_{N}}{1-\delta_{N}}<2, \quad r_{N}=b_{N} \eta_{N}^{(0)} G_{N} \leq R,
$$


where

$$
\begin{aligned}
b_{N} & =b /\left(1-b \rho_{N}\right), \\
\rho_{N} & =\alpha_{N}^{\prime}+\beta_{N}^{\prime}+L^{\prime} \delta_{N} /\left(1-\delta_{N}\right)+L \delta /(1-\delta), \\
G_{N} & =H_{N}+\sum_{m=N}^{\infty}\left(h_{N}^{(0)} / 2\right)^{S_{m}}<2 H_{N}, \\
s_{m} & =\sum_{i=N}^{m}\left(k_{i}-1\right), \\
H_{N}=1 /\left(1-h_{N}^{(0)} / 2\right), &
\end{aligned}
$$

then equation (1.1) has in the ball $S\left(u_{N}^{(0)}, r_{N}\right) \subset X$ a solution $u^{*}$ to which the projection-iteration process of approximations (3.1) converges with the error estimate

$$
\left\|u_{n}^{\left(k_{n}\right)}-u^{*}\right\| \leq b_{N} \eta_{N}^{(0)} V_{n}\left(h_{N}^{(0)} / 2\right)^{S_{n}}, \quad n \geq N
$$

where $V_{n}=H_{N}+\sum_{m=n+1}^{\infty}\left(h_{N}^{(0)} / 2\right)^{S_{m}-S_{n}}<2 H_{N}$.

Proof. First of all, we note that the second condition in (2.6) implies the existence of bounded inverse operator $[D(u)]^{-1}, u \in S\left(u_{N}^{(0)}, R\right)$; while taking into account (3.2) the estimate $\left\|[D(u)]^{-1}\right\|_{X \rightarrow X} \leq L /(1-\delta)$ holds for all $u \in S\left(u_{N}^{(0)}, R\right)$. Similarly, from the conditions (2.7) and (2.4) there follows the existence of bounded inverse operators $\left[D_{n}\left(u_{n}\right)\right]^{-1}, u_{n} \in \Omega_{n}$ with the norm $\left\|\left[D_{n}\left(u_{n}\right)\right]^{-1}\right\|_{X_{n} \rightarrow X_{n}} \leq$ $L^{\prime} /\left(1-\delta_{n}\right), n \geq N$. Further, based on the first condition (2.6) and the proximity conditions (2.1), (2.2) the existence of operators $D_{n}\left(u_{n}\right)$ implies their boundedness, beginning with some $n=N_{1} \geq N$. Indeed, since for $u_{n} \in \Omega_{n}, z_{n} \in X_{n}$

$$
\begin{aligned}
& \left\|\left[D_{n}\left(u_{n}\right)\right]^{-1} z_{n}-\left[D\left(u_{n}\right)\right]^{-1} z_{n}\right\| \leq\left(\left\|\left[D_{n}\left(u_{n}\right)\right]^{-1}-A_{n}^{\prime}\left(u_{n}\right)\right\|_{X_{n} \rightarrow X_{n}}\right. \\
& \quad+\left\|A_{n}^{\prime}\left(u_{n}\right)-P_{n} A^{\prime}\left(u_{n}\right)\right\|_{X_{n} \rightarrow X_{n}}+\left\|P_{n} A^{\prime}\left(u_{n}\right)-A^{\prime}\left(u_{n}\right)\right\|_{X \rightarrow X} \\
& \left.\quad+\left\|A^{\prime}\left(u_{n}\right)-\left[D\left(u_{n}\right)\right]^{-1}\right\|_{X \rightarrow X}\right)\left\|z_{n}\right\| \leq \rho_{n}\left\|z_{n}\right\|,
\end{aligned}
$$

where $\rho_{n}=L^{\prime} \delta_{n} /\left(1-\delta_{n}\right)+\alpha_{n}^{\prime}+\beta_{n}^{\prime}+L \delta /(1-\delta)$, then

$$
\begin{aligned}
\left\|\left[D_{n}\left(u_{n}\right)\right]^{-1} z_{n}\right\| & \geq\left\|\left[D\left(u_{n}\right)\right]^{-1} z_{n}\right\|-\left\|\left[D_{n}\left(u_{n}\right)\right]^{-1} z_{n}-\left[D\left(u_{n}\right)\right]^{-1} z_{n}\right\| \\
& \geq\left(1-b \rho_{n}\right) / b\left\|z_{n}\right\|,
\end{aligned}
$$

and since under the conditions of the theorem $b \rho_{n}<1$ for $n \geq N_{1}$, then for these numbers $n$ we will have

$$
\left\|D_{n}\left(u_{n}\right)\right\|_{X_{n} \rightarrow X_{n}} \leq b_{n}=b /\left(1-b \rho_{n}\right), \quad u_{n} \in \Omega_{n} .
$$

Let us prove the feasibility of process (3.1). Note that the possibility of replacing equations (1.2) by linearized equations

$$
A_{n} u_{n}^{(k)}+\left[D_{n}\left(u_{n}^{(k)}\right)\right]^{-1}\left(u_{n}-u_{n}^{(k)}\right)=f_{n}, \quad k=0,1, \ldots ; n \geq N
$$


respectively follows from the existence of continuous operators $\left[D_{n}\left(u_{n}\right)\right]^{-1}$ close to $A_{n}^{\prime}\left(u_{n}\right), u_{n} \in \Omega_{n}$ for the specified $n$. We establish (by mathematical induction) that all subsequent approximations $u_{n}^{(0)}$ for $n>N$ have the same properties (2.8), (3.3) and that they belong to the ball $S\left(u_{N}^{(0)}, r_{N}\right) \subset X$. Based on the theorem conditions, it can be shown that for $n=N, N+1, \ldots, m$

$$
\left\|A_{n} u_{n}^{(0)}-f_{n}\right\| \leq \eta_{n}^{(0)}, \quad h_{n}^{(0)}=b_{n}^{2} L^{\prime} \eta_{n}^{(0)}+\frac{2 b_{n} L^{\prime} \delta_{n}}{1-\delta_{n}}<2 .
$$

In addition, as it follows from the proof of Theorem 2 of [6], at any fixed $n$ $(N \leq n \leq m)$ the conditions

$$
\left\|A_{n} u_{n}^{(k)}-f_{n}\right\| \leq \eta_{n}^{(k)}, \quad h_{n}^{(k)}=b_{n}^{2} L^{\prime} \eta_{n}^{(k)}+\frac{2 b_{n} L^{\prime} \delta_{n}}{1-\delta_{n}}<2
$$

hold for each number $k=1,2, \ldots, k_{n}$. We show the feasibility of (3.6) for $n=m+1$. Insofar as

$$
\left\|A_{m+1} u_{m+1}^{(0)}-f_{m+1}\right\| \leq\left\|A_{m+1} u_{m+1}^{(0)}-A_{m} u_{m+1}^{(0)}\right\|+\left\|A_{m} u_{m}^{\left(k_{m}\right)}-f_{m}\right\|+\left\|f_{m}-f_{m+1}\right\|,
$$

then based on the proximity conditions (2.1)-(2.3) from the relations

$$
\begin{aligned}
\| A_{m+1} u_{m+1}^{(0)} & -A_{m} u_{m+1}^{(0)}\|\leq\| A_{m+1} u_{m+1}^{(0)}-P_{m+1} A u_{m+1}^{(0)} \| \\
& +\left\|P_{m+1} A u_{m+1}^{(0)}-A u_{m+1}^{(0)}\right\|+\left\|A u_{m}^{\left(k_{m}\right)}-P_{m} A u_{m}^{\left(k_{m}\right)}\right\| \\
& +\left\|P_{m} A u_{m}^{\left(k_{m}\right)}-A_{m} u_{m}^{\left(k_{m}\right)}\right\| \leq \alpha_{m+1}+\beta_{m+1}+\beta_{m}+\alpha_{m}
\end{aligned}
$$

and from the first of the conditions (3.7) we obtain:

$$
\left\|A_{m+1} u_{m+1}^{(0)}-f_{m+1}\right\| \leq \theta_{m}+\eta_{m}^{\left(k_{m}\right)}=\eta_{m+1}^{(0)},
$$

where $\theta_{m}=\alpha_{m}+\alpha_{m+1}+\beta_{m}+\beta_{m+1}+\gamma_{m}+\gamma_{m+1}$, that is, the first of the conditions (3.6) for $n=m+1$ holds true. Let us show the fulfillment of the second one.

Proof of the Theorem 2 of [6] implies that for any $k=0,1, \ldots, k_{m}-1$

$$
\begin{aligned}
\left\|A_{m} u_{m}^{(k+1)}-f_{m}\right\| & =\left\|A_{m} u_{m}^{(k+1)}-A_{m} u_{m}^{(k)}-\left[D_{m}\left(u_{m}^{(k)}\right)\right]^{-1}\left(u_{m}^{(k+1)}-u_{m}^{(k)}\right)\right\| \\
& \leq \frac{L^{\prime}}{2}\left\|u_{m}^{(k+1)}-u_{m}^{(k)}\right\|^{2}+\frac{L^{\prime} \delta_{m}}{1-\delta_{m}}\left\|u_{m}^{(k+1)}-u_{m}^{(k)}\right\| \\
& \leq \frac{L^{\prime}}{2} b_{m}^{2} \eta_{m}^{(k)^{2}}+\frac{L^{\prime} \delta_{m}}{1-\delta_{m}} b_{m} \eta_{m}^{(k)}=\frac{h_{m}^{(k)}}{2} \eta_{m}^{(k)}=\eta_{m}^{(k+1)},
\end{aligned}
$$

so

$$
\eta_{m}^{\left(k_{m}\right)}=\frac{h_{m}^{\left(k_{m}-1\right)}}{2} \eta_{m}^{\left(k_{m}-1\right)}=\cdots=\frac{1}{2^{k_{m}}} h_{m}^{\left(k_{m}-1\right)} h_{m}^{\left(k_{m}-2\right)} \ldots h_{m}^{(0)} \eta_{m}^{(0)}
$$


Because in (3.8) $\theta_{m} \rightarrow 0$ when $m \rightarrow \infty$ and because by virtue of (3.7) $h_{m}^{\left(k_{m}-1\right)}<2$, there exists a number $m=N_{2} \geq N$ beginning with which

$$
\eta_{m+1}^{(0)} \leq \frac{1}{2^{k_{m}-1}} h_{m}^{\left(k_{m}-2\right)} h_{m}^{\left(k_{m}-3\right)} \ldots h_{m}^{(0)} \eta_{m}^{(0)}
$$

Since, obviously $b_{m+1} \leq b_{m}, \delta_{m+1} \leq \delta_{m}$, then taking into account (3.10) and (3.7) we have for all $m \geq N_{2}$ :

$$
\begin{aligned}
h_{m+1}^{(0)} & =b_{m+1}^{2} L^{\prime} \eta_{m+1}^{(0)}+\frac{2 b_{m+1} L^{\prime} \delta_{m+1}}{1-\delta_{m+1}} \\
& \leq b_{m}^{2} L^{\prime} \frac{1}{2^{k_{m}-1}} h_{m}^{\left(k_{m}-2\right)} h_{m}^{\left(k_{m}-3\right)} \ldots h_{m}^{(0)} \eta_{m}^{(0)}+\frac{2 b_{m} L^{\prime} \delta_{m}}{1-\delta_{m}} \\
& =b_{m}^{2} L^{\prime} \eta_{m}^{\left(k_{m}-1\right)}+\frac{2 b_{m} L^{\prime} \delta_{m}}{1-\delta_{m}}=h_{m}^{\left(k_{m}-1\right)}<2,
\end{aligned}
$$

that is, the second of the conditions (3.6) for $n=m+1$ also holds true.

Let number $N:=\max \left\{N_{1}, N_{2}\right\}$ be the initial one in formulas (3.1).

Let's show that the approximations $u_{n+1}^{(0)}$ belong to the ball $S\left(u_{N}^{(0)}, r_{N}\right) \subset X$ for all $n \geq N$. It's obvious that

$$
\left\|u_{n+1}^{(0)}-u_{N}^{(0)}\right\| \leq \sum_{m=N}^{n}\left\|u_{m+1}^{(0)}-u_{m}^{(0)}\right\|, \quad n \geq N ;
$$

in turn, for each $m=N, N+1, \ldots, n$

$$
\left\|u_{m+1}^{(0)}-u_{m}^{(0)}\right\|=\left\|u_{m}^{\left(k_{m}\right)}-u_{m}^{(0)}\right\| \leq \sum_{k=0}^{k_{m}-1}\left\|u_{m}^{(k+1)}-u_{m}^{(k)}\right\| .
$$

Based on formulas (3.1), (3.5), (3.7), (3.9) for any numbers $m=N, N+1, \ldots, n$ and $k=0,1, \ldots, k_{m}-1$ we obtain:

$$
\begin{aligned}
\left\|u_{m}^{(k+1)}-u_{m}^{(k)}\right\| & \leq\left\|D_{m}\left(u_{m}^{(k)}\right)\right\|_{X_{m} \rightarrow X_{m}}\left\|A_{m} u_{m}^{(k)}-f_{m}\right\| \leq b_{m} \eta_{m}^{(k)} \\
& =b_{m} \frac{1}{2^{k}} h_{m}^{(k-1)} h_{m}^{(k-2)} \ldots h_{m}^{(0)} \eta_{m}^{(0)},
\end{aligned}
$$

and because of

$$
\begin{aligned}
h_{m}^{(k+1)} & =b_{m}^{2} L^{\prime} \eta_{m}^{(k+1)}+\frac{2 b_{m} L^{\prime} \delta_{m}}{1-\delta_{m}} \\
& =b_{m}^{2} L^{\prime} \frac{h_{m}^{(k)}}{2} \eta_{m}^{(k)}+\frac{2 b_{m} L^{\prime} \delta_{m}}{1-\delta_{m}} \\
& <b_{m}^{2} L^{\prime} \eta_{m}^{(k)}+\frac{2 b_{m} L^{\prime} \delta_{m}}{1-\delta_{m}} \\
& =h_{m}^{(k)}<2, \quad k=0,1, \ldots, k_{m}-1,
\end{aligned}
$$


we have

$$
\left\|u_{m}^{(k+1)}-u_{m}^{(k)}\right\| \leq b_{m}\left(h_{m}^{(0)} / 2\right)^{k} \eta_{m}^{(0)}, \quad k=0,1, \ldots, k_{m}-1 .
$$

Let's evaluate here $\eta_{m}^{(0)}$ and $h_{m}^{(0)}(N+1 \leq m \leq n)$ through $\eta_{N}^{(0)}$ and $h_{N}^{(0)}$. Applying (3.12) in formulas (3.10) and (3.11), we obtain the relations

$$
\begin{gathered}
\eta_{m+1}^{(0)}<\left(h_{m}^{(0)} / 2\right)^{k_{m}-1} \eta_{m}^{(0)} ; \\
h_{m+1}^{(0)} \leq h_{m}^{\left(k_{m}-1\right)}<h_{m}^{(0)} \leq h_{m-1}^{\left(k_{m-1}-1\right)}<h_{m-1}^{(0)} \leq \cdots<h_{N}^{(0)}, \quad m \geq N,
\end{gathered}
$$

which implies that

$$
\begin{aligned}
\eta_{m}^{(0)} & <\left(h_{m-1}^{(0)} / 2\right)^{k_{m-1}-1} \eta_{m-1}^{(0)} \\
& <\left(h_{m-1}^{(0)} / 2\right)^{k_{m-1}-1}\left(h_{m-2}^{(0)} / 2\right)^{k_{m-2}-1} \eta_{m-2}^{(0)}<\ldots \\
& <\left(h_{N}^{(0)} / 2\right)^{S_{m-1}} \eta_{N}^{(0)}
\end{aligned}
$$

where $s_{m-1}=\sum_{i=N}^{m-1}\left(k_{i}-1\right), m=N+1, N+2, \ldots, n$. With this in mind

$$
\begin{gathered}
\left\|u_{m}^{(k+1)}-u_{m}^{(k)}\right\| \leq b_{N}\left(h_{N}^{(0)} / 2\right)^{S_{m-1}+k} \eta_{N}^{(0)}, \\
k=0,1, \ldots, k_{m}-1 ; \quad m=N+1, N+2, \ldots, n ; \\
\left\|u_{N}^{(k+1)}-u_{N}^{(k)}\right\| \leq b_{N}\left(h_{N}^{(0)} / 2\right)^{k} \eta_{N}^{(0)}, \quad k=0,1, \ldots, k_{N}-1,
\end{gathered}
$$

so

$$
\begin{aligned}
\left\|u_{n+1}^{(0)}-u_{N}^{(0)}\right\| & \leq \sum_{m=N}^{n} \sum_{k=0}^{k_{m}-1}\left\|u_{m}^{(k+1)}-u_{m}^{(k)}\right\| \\
& \leq b_{N} \eta_{N}^{(0)}\left[\sum_{k=0}^{k_{N}-1}\left(h_{N}^{(0)} / 2\right)^{k}+\sum_{m=N+1}^{n} \sum_{k=0}^{k_{m}-1}\left(h_{N}^{(0)} / 2\right)^{S_{m-1}+k}\right] \\
& =b_{N} \eta_{N}^{(0)}\left[\sum_{k=0}^{S_{n}}\left(h_{N}^{(0)} / 2\right)^{k}+\sum_{m=N}^{n-1}\left(h_{N}^{(0)} / 2\right)^{S_{m}}\right] \\
& <b_{N} \eta_{N}^{(0)} G_{N}=r_{N}, \quad n \geq N
\end{aligned}
$$

that is, each $u_{n+1}^{(0)}$ where $n \geq N$ (and also all $u_{n}^{(k)}\left(k=1,2, \ldots, k_{n}\right)$ by virtue of the Theorem 2 from [6]) belong to the ball $S\left(u_{N}^{(0)}, r_{N}\right)$. Thus, the feasibility of process (3.1) is proved.

Let's now show that the sequence $\left\{u_{n}^{\left(k_{n}\right)}\right\}_{n=N}^{\infty}$, which is determined by formulas (3.1), converges in $S\left(u_{N}^{(0)}, r_{N}\right)$. Using (3.13) for any numbers $n \geq N$ and $p \in \mathbb{N}$ 
we write:

$$
\begin{aligned}
\left\|u_{n+p}^{\left(k_{n+p}\right)}-u_{n}^{\left(k_{n}\right)}\right\| & \leq \sum_{m=n+1}^{n+p}\left\|u_{m}^{\left(k_{m}\right)}-u_{m-1}^{\left(k_{m-1}\right)}\right\|=\sum_{m=n+1}^{n+p}\left\|u_{m}^{\left(k_{m}\right)}-u_{m}^{(0)}\right\| \\
& \leq \sum_{m=n+1}^{n+p} \sum_{k=0}^{k_{m}-1}\left\|u_{m}^{(k+1)}-u_{m}^{(k)}\right\| \\
& \leq b_{N} \eta_{N}^{(0)} \sum_{m=n+1}^{n+p} \sum_{k=0}^{k_{m}-1}\left(h_{N}^{(0)} / 2\right)^{S_{m-1}+k} \\
& =b_{N} \eta_{N}^{(0)}\left[\sum_{k=0}^{k_{n+1}-1}\left(h_{N}^{(0)} / 2\right)^{S_{n}+k}+\sum_{m=n+2}^{n+p} \sum_{k=0}^{k_{m}-1}\left(h_{N}^{(0)} / 2\right)^{S_{m-1}+k}\right] \\
& \left.=b_{N} \eta_{N}^{(0)}\left(h_{N}^{(0)} / 2\right)^{S_{n}}\left[\sum_{k=0}^{n+p-1}\left(h_{N}^{(0)} / 2\right)^{k}+\sum_{m=n+1}^{S_{n+p}-S_{n}} / 2\right)^{S_{m}-S_{n}}\right] \\
& <b_{N} \eta_{N}^{(0)}\left(h_{N}^{(0)} / 2\right)^{S_{n}} 2 H_{N} .
\end{aligned}
$$

Since $h_{N}^{(0)}<2$, then $\left\|u_{n+p}^{\left(k_{n+p}\right)}-u_{n}^{\left(k_{n}\right)}\right\| \rightarrow 0$ when $n \rightarrow \infty$, that means the fundamentality of the sequence $\left\{u_{n}^{\left(k_{n}\right)}\right\}_{n=N}^{\infty} \subset S\left(u_{N}^{(0)}, r_{N}\right)$. By virtue of the completeness of the space $X$, there exists an element $u^{*} \in S\left(u_{N}^{(0)}, r_{N}\right)$ such that $u^{*}=\lim _{n \rightarrow \infty} u_{n}^{\left(k_{n}\right)}$. Passing to the limit at $p \rightarrow \infty$ in (3.14) and denoting

$$
\begin{aligned}
V_{n} & =\lim _{p \rightarrow \infty}\left[\sum_{k=0}^{S_{n+p}-S_{n}}\left(h_{N}^{(0)} / 2\right)^{k}+\sum_{m=n+1}^{n+p-1}\left(h_{N}^{(0)} / 2\right)^{S_{m}-S_{n}}\right] \\
& =\sum_{k=0}^{\infty}\left(h_{N}^{(0)} / 2\right)^{k}+\sum_{m=n+1}^{\infty}\left(h_{N}^{(0)} / 2\right)^{S_{m}-S_{n}}, \quad n \geq N,
\end{aligned}
$$

we obtain the error estimate (3.4).

To prove that the limit $u^{*}$ of the sequence $\left\{u_{n}^{\left(k_{n}\right)}\right\}_{n=N}^{\infty}$ is a solution of equation (1.1), we consider the residual of method (3.1) on the $n$-th step $(n \geq N)$ :

$$
\begin{aligned}
\left\|A u_{n}^{\left(k_{n}\right)}-f\right\| & \leq\left\|A u_{n+1}^{(0)}-P_{n+1} A u_{n+1}^{(0)}\right\|+\left\|P_{n+1} A u_{n+1}^{(0)}-A_{n+1} u_{n+1}^{(0)}\right\| \\
& +\left\|A_{n+1} u_{n+1}^{(0)}-f_{n+1}\right\|+\left\|f_{n+1}-f\right\| \leq \beta_{n+1}+\alpha_{n+1}+\eta_{n+1}^{(0)}+\gamma_{n+1} .
\end{aligned}
$$

Since, $\alpha_{n+1}, \beta_{n+1}, \gamma_{n+1}, \eta_{n+1}^{(0)} \rightarrow 0$ when $n \rightarrow \infty$, and since the operator $A$ is continuous due to Fréchet differentiability, then by tending $n \rightarrow \infty$ in the last inequality, we obtain that $A u^{*}=f$. The theorem is proved.

Note that the projection-iteration implementation (3.1) of the Newton-like method generally converges more slowly than the projection-iteration process $(2.5)$ based on the classical Newton's method. An exception is the case, when $\delta=0$, 
$\delta_{n}=0(n \geq N)$ in formulas (2.6), (2.7), that leads to the transformation of method (3.1) into (2.5); in such a situation, the error estimate (3.4) for method (3.1) (or, equivalently, method (2.5)) is significantly overestimated, and for this case the more appropriate result is contained in Theorem 2.1.

For equation (1.1) under the Theorem 2.1 conditions, along with the projection-iteration method (2.5) based on the Newton's method, one can consider the approximation process based on the modified Newton's method:

$$
\begin{gathered}
u_{n}^{(k+1)}=u_{n}^{(k)}-\left[A_{n}^{\prime}\left(u_{n}^{(0)}\right)\right]^{-1}\left(A_{n} u_{n}^{(k)}-f_{n}\right), \quad k=0,1, \ldots, k_{n}-1 ; \\
u_{n+1}^{(0)}=u_{n}^{\left(k_{n}\right)}, \quad n \geq N ; \quad u_{N}^{(0)} \in \Omega_{N} \subset X,
\end{gathered}
$$

and under the Theorem 3.1 conditions, along with the projection-iteration method (3.1), one can consider the approximation process based on the modified Newtonlike method:

$$
\begin{gathered}
u_{n}^{(k+1)}=u_{n}^{(k)}-D_{n}\left(u_{n}^{(0)}\right)\left(A_{n} u_{n}^{(k)}-f_{n}\right), \quad k=0,1, \ldots, k_{n}-1 ; \\
u_{n+1}^{(0)}=u_{n}^{\left(k_{n}\right)}, \quad n \geq N ; \quad u_{N}^{(0)} \in \Omega_{N} \subset X .
\end{gathered}
$$

Such the projection-iteration processes (although they converge more slowly than the process (2.5) based on the Newton's method) are less laborious, since for each $n \geq N$ they use operators $\left[A_{n}^{\prime}\left(u_{n}^{(0)}\right)\right]^{-1}$ or $D_{n}\left(u_{n}^{(0)}\right)$ which correspond only to the initial point $u_{n}^{(0)} \in \Omega_{n}$, and this obviously leads to a computational overhead reduction in numerical implementation.

We note, finally, that while solving nonlinear operator equations of the form (1.1), as follows from the proofs of Theorems 2.1, 3.1 on the convergence of projection-iteration methods based on the Newton's method and the Newton-like one respectively, the convergence of corresponding sequences $\left\{u_{n}^{\left(k_{n}\right)}\right\}_{n=N}^{\infty}$ (when $n \rightarrow \infty)$ towards an exact solution $u^{*}$ in $X$ occurs under an arbitrary choice of numbers $k_{n}$. However, to prevent a sharp increase with increasing $n$ of amount of computations needed to find the next approximation, we have to consider a problem of the appropriate choice of numbers $k_{n}$ at each $n \geq N$. Some recommendations on this issue have been given in [2]. In particular, there has been considered a way to choose numbers $k_{n}$ so that the element $u_{n}^{\left(k_{n}\right)}$ would be a good initial approximation for the $(n+1)$-th approximate equation of the form (1.2), that is, that the residual $A_{n+1} u_{n+1}^{(0)}-f_{n+1}$ would have, if possible, a small value. The idea underlying this way to choose numbers $k_{n}$ also makes it possible to determine the most acceptable number $n+p(p \geq 1)$ of the approximate equation following the $n$-th one in the sequence of equations (1.2). Some other ways to choose numbers $k_{n}$ in projection-iteration methods of solving nonlinear equations as well as their application in solving specific problems, can be found in [3,7,9-11].

\section{References}

1. I. K. ARgYros, On Newton's method for solving nonlinear equations and function splitting, Numerical Mathematics: Theory, Methods and Applications, 4(1)(2011), $53-67$. 
2. S. D. Balashova, Approximate methods for solving operator equations, DSU, Dnipropetrovsk, 1980 (in Russian).

3. S. D. BAlashova, L. L. HART, On one approach to solving elliptic nonlinear problems, Mathematical models and computational methods in applied problems, Dnipropetrovsk: DSU, 1996, 24-29 (in Russian).

4. P. Deuflhard, Newton methods for nonlinear problems: affine invariance and adaptive algorithms, Springer Series in Computational Mathematics, Vol. 35, Springer-Verlag, Berlin, 2004.

5. J. A. Ezquerro Fernández, M. Á. Hernández Verón, Newton's Method: an Updated Approach of Kantorovich's Theory, Birkhäuser, Cham, Switzerland, 2017.

6. L. L. HART, Iterative Newton-like processes for solving nonlinear operator equations and their modifications, Problems of applied mathematics and mathematical modeling, Dnipropetrovsk: Lira, 17 (2017), 32-41 (in Ukrainian).

7. L. L. HART, On the further realization of the projection-iterative principle of solving the optimization problem for a dynamic system, Problems of applied mathematics and mathematical modeling, Dnipropetrovsk: DNU, 2 (2002), 42-48 (in Ukrainian).

8. L. L. HART, Projection-iteration methods for solving operator equations and infinite dimensional optimization problems, The thesis for the degree of Doctor of Physical and Mathematical Sciences, Dnipro: DNU, 2016 (in Ukrainian).

9. L. L. HART, The application of projection-iteration methods to solving optimal control problems for systems of ordinary differential equations, Hamburger Beiträge zur Angewandten Mathematik, Reihe A, Universität Hamburg, Deutschland, 152 (2000), 1-17.

10. L. L. Hart, N. V. Polyakov, Application of a projection-iterative approach based on the method of lines to solving a nonlinear elliptic boundary value problem, Problems of applied mathematics and mathematical modeling, Dnipropetrovsk: DNU, 5 (2005), 68-77 (in Ukrainian).

11. L. L. Hart, N. V. Polyakov, Projection-iteration realization on the NewtonKantorovich method for solving nonlinear integral equations, Journal of Automation and Information Sciences, 44 (1)(2012), 40-49.

12. L. V. Kantorovich, Functional analysis and applied mathematics, Uspekhi Mat. Nauk, 3 (1948), 89-185 (in Russian).

13. L. V. Kantorovich, On Newton's method for functional equations, Doklady Akademii Nauk SSSR, 59 (7)(1948), 1237-1240 (in Russian).

14. L. V. Kantorovich, G. P. Akilov, Functional Analysis, Nevsky Dialect, St. Petersburg, 2004 (in Russian).

15. I. P. MYsovskin, On the convergence of the method of L. V. Kantorovich for the solution of nonlinear functional equations and its applications, Vestnik Leningrad Univ., 11 (1953), 25-48 (in Russian).

16. B. T. POLYAK, Newton-Kantorovich method and its global convergence, Journal of Mathematical Sciences, 133 (4)(2006), 1513-1523 (in Russian).

17. B. A. Vertgeim, On conditions for the applicability of Newton's method, Doklady Akademii Nauk SSSR, 110 (5)(1956), 719-722 (in Russian).

18. T. Yамамото, Historical developments in convergence analysis for Newton's and Newton-like methods, Journal of Computational and Applied Mathematics, 124 (1-2)(2000), 1-23. 